\title{
500 Jahre Reformation (1517-2017) - gemeinsam ein Christusfest feiern? \\ Systematisch-theologische Reflexionen in ökumenischer und internationaler Perspektive
}

\author{
Margit Eckholt \\ INSTITUT FÜR KATHOLISCHE THEOLOGIE \\ UNIVERSITÄT OSNABRÜCK \\ Margit.Eckholt@uni-osnabrueck.de
}

Zusammenfassung: Am 31. Oktober 2017 jährt sich der Thesenanschlag Martin Luthers an der Schlosskirche in Wittenberg zum 500. Mal. Das von einer lutherisch-katholischen Dialogkommission erarbeitete Dokument "Vom Konflikt zur Gemeinschaft. Gemeinsames lutherischkatholisches Reformationsgedenken 2017" macht deutlich, dass heute nur ein gemeinsames Erinnern an die Reformation - ein "Gedenken", kein "Jubiläum" - möglich ist und dass ein solches in einem internationalen Horizont erfolgen muss. Der vorliegende Aufsatz erarbeitet in systematischtheologischer Perspektive einen hermeneutischen Rahmen für dieses ökumenische und internationale Gedenken der Reformation und geht auf zentrale Thesen der Dokumente "Vom Konflikt zur Gemeinschaft" und "Erinnerung heilen - Jesus Christus bezeugen" ein.

Schlüsselwörter: Luther, Reformationsgedenken, Rechtfertigung, Erinnerung und Versöhnung, Ökumene, interkulturelle Theologie

Resumen: El 31 de octubre 2017 se hará memoria de los 500 años de las tesis de Martin Lutero, puestas en la iglesia del castillo en Wittenberg. El documento „Del conflicto a la comunión“ explica que hoy sólamente una memoria en común entre la Iglesia católica y las Iglesias protestantes es posible, y eso no es un "jubileo", pero sí una memoria en un horizonte internacional. El artículo elabora el marco hermenéutico para esa memoria ecuménica y internacional, en una perspectiva de teología sistemática. Se analizan las tesis centrales de los documentos "Del conflicto a la communion" y "Sanar la memoria - dar testimonio de Jesucristo".

Palabras clave: Lutero, recuerdo de la Reforma, justificación, memoria y reconciliación, ecumenismo, teología intercultural. 
1. "Die Geschichte anders erzÄHLen": Ein Reformationsgedenken in ÖKUMENISCHER UND INTERNATIONALER PERSPEKTIVE

Am 17. Juni 2013 haben Kardinal Kurt Koch, der Vorsitzende des Päpstlichen Rates für die Einheit der Christen, und Martin Junge, der Generalsekretär des Lutherischen Weltbundes, in Genf das von einer lutherisch-katholischen Dialogkommission erarbeitete Dokument "Vom Konflikt zur Gemeinschaft. Gemeinsames lutherisch-katholisches Reformationsgedenken 2017" vorgestellt, das wichtige Akzente setzt für den Weg auf das 500-jährige Gedenken der Reformation im Jahr 2017 hin. Sie haben deutlich gemacht, dass heute nur ein gemeinsames Erinnern an die Reformation - ein "Gedenken", kein "Jubiläum" - möglich ist und dass ein solches in einem internationalen Horizont erfolgen muss.

"Es ist das erste Reformationsgedenken, das im Zeitalter der Ökumene stattfindet. So bietet das gemeinsame Gedenken die Gelegenheit, die Gemeinschaft zwischen Katholiken und Lutheranern zu vertiefen. Es ist das erste Reformationsgedenken im Zeitalter der Globalisierung. Darum muss das gemeinsame Gedenken die Erfahrungen und Perspektiven der Christen aus dem Süden und Norden, aus dem Osten und Westen einbeziehen" (Nr. 4, S. 13).

Das sind zwei Perspektiven für die Erinnerung an das Ereignis der Reformation, die sowohl für die Kirchen der Reformation als auch die katholische Kirche eine neue Herausforderung darstellen und die aus theologischer Perspektive weiter aufzuarbeiten sind: der gemeinsame Horizont des Gedenkens auf der einen und der internationale Horizont auf der anderen Seite, und die beide darin verbunden sind, als Christen und Christinnen "in einer Zeit, die durch eine wachsende Zahl neuer religiöser Bewegungen und zugleich durch die Zunahme der Säkularisierung an vielen Orten gekennzeichnet ist" (Nr. 4, S. 13) auf neue Weise ein "gemeinsames Glaubenszeugnis" abzulegen. Das Christentum ist in den letzten Jahrzehnten weiter gewachsen; von ca. 7,4 Milliarden Menschen auf der Erde sind ca. 32 \% Christen und Christinnen; 2010 lebten 285 Millionen (= 23,8 \%) Katholiken in Europa, 90 Millionen

\footnotetext{
Vom Konflikt zur Gemeinschaft: Gemeinsames lutherisch-katholisches Reformationsgedenken im Jahr 2017: Bericht der Lutherisch/Römisch-katholischen Kommission für die Einheit (Evangelische Verlagsanstalt, Leipzig/Bonifatius, Paderborn 2013). Das Dokument wird mit Nummer und Seitenzahl im Text zitiert.
} 
(7,5 \%) in Nordamerika, 496 Millionen $(41,5 \%)$ in Lateinamerika, 140 Millionen (11,7\%) in Asien und Ozeanien und in Afrika 185 Millionen $(15,5 \%)$. Europa und Nordamerika repräsentieren inzwischen also nicht einmal mehr ein Drittel aller Gläubigen weltweit. ${ }^{2}$ In den afrikanischen und asiatischen Ländern, in denen das Christentum weiter wächst, leben Christen und Christinnen in einem multireligiösen Umfeld, und gerade hier ist dieses "gemeinsame Glaubenszeugnis" von größter Bedeutung: die "Feindseligkeit konfessioneller Gegensätze", so das Dokument "Vom Konflikt zur Gemeinschaft", fügt der "Glaubwürdigkeit der Christen" Schaden zu (Nr. 15. S. 17). "Die Art und Weise, wie Christen mit Unterschieden untereinander umgehen, kann Menschen anderer Religionen etwas über ihren Glauben offenbaren" (ebd.). In einer globalisierten Welt, die zu einer "Weltunordnung" zu werden droht, "in der religiös-kulturelle Unterschiede politisch instrumentalisiert werden und zu unterhörten Gewaltausbrüchen führen”, so Kardinal Walter Kasper in seinen Überlegungen zu Martin Luther in ökumenischer Perspektive 3 , müssen Christen und Christinnen gemeinsam "der brutalen Gewalt, die sich oft ein religiöses Mäntelchen umhängt, die universale christliche Botschaft der Liebe und den gewaltlosen Einsatz für Gerechtigkeit, Frieden und Freiheit entgegensetzen"“. "Die beste ökumenische Idee für das Jahr 2017", so Kardinal Kasper, "ist darum die, ein gemeinsames Christusfest zu feiern", und hier bezieht er sich auf den Impuls des Vorsitzenden der Evangelischen Kirche in Deutschland, Bischof Heinrich Bedford-Strohm, und das Dokument von EKD und DBK (Deutscher Bischofskonferenz) "Erinnerung heilen - Jesus Christus bezeugen" , das am 16. September 2016 im unmittelbaren Vorfeld des Gedenkjahres veröffentlicht worden ist. "Das wäre", so Kasper,

2 Vgl. dazu Johannes Meier, Die Wirkungen des II. Vatikanischen Konzilsin Lateinamerika, Asien und Afrika. 1965-2015, in: Ausgabe 4 / 2015 der Zeitschrift „Salve“ 4/2015, 73-85; vgl. auch John Allen, Das neue Gesicht der Kirche. Die Zukunft des Katholizismus, Gütersloh 2010; Philipp Jenkins, Das Christentum der Zukunft wird vom Süden bestimmt, http://www.der-eberblick.de/ueberblick. archiv/one.ueberblick.article/ueberblick28f1.html?entry=page.200303.080

3 W. Kasper, Martin Luther: Eine ökumenische Perspektive (Grünewald, Ostfildern 22016) 55.

4 W. KASPER, Martin Luther..., 56.

5 Erinnerung beilen - Jesus Christus bezengen: Ein gemeinsames Wort zum Jahr 2017, 16.09.2016, editado por Evangelische Kirche in Deutschland y por el Sekretariat der Deutschen Bischofskonferenz (Hannover/Bonn 2016). 
"ökumenische Katholizität ganz nah bei den Menschen und mitten in der Welt".

Grundlage dieses gemeinsamen Erinnerns ist ein "anderer" Blick auf die Geschichte und mit ihm die "Entdeckung der anderen", die Teil dieser Geschichte sind, jedoch ausgeblendet, marginalisiert und als "Nicht-Rechtgläubige" "verketzert" wurden. "Was in der Vergangenheit geschehen ist, kann nicht geändert werden", so formuliert das Dokument „Vom Konflikt zur Gemeinschaft“ im grundlegenden hermeneutisch-methodischen Kapitel zu "neuen Perspektiven" auf "Martin Luther und die Reformation", und dann heißt es:

"Was jedoch von der Vergangenheit erinnert wird und wie das geschieht, kann sich im Lauf der Zeit tatsächlich verändern. Erinnerung macht die Vergangenheit gegenwärtig. Während die Vergangenheit selbst unveränderlich ist, ist die Präsenz der Vergangenheit in der Gegenwart veränderlich. Mit Blick auf 2017 geht es nicht darum, eine andere Geschichte zu erzählen, sondern darum, diese Geschichte anders zu erzählen" (Nr. 16, S. 18)).

Dieses "anders erzählen" - in das auch die Überlegungen des vorliegenden Beitrags eingeschrieben sind - gründet in den ökumenischen Dialogprozessen seit den 80er Jahren letzten Jahrhunderts, der "katholischen Anerkennung des Augsburger Bekenntnisses" (Nr. 24, S. 21), d.h. dem Bekenntnis der lutherischen Reichsstände am 25. Juni 1530 in Augsburg, einer der zentralen Bekenntnisschriften der Kirchen der lutherischen und reformierten Kirchen, die auch Grundlage für den Augsburger Religionsfrieden (25. September 1555) war, eine Einigung, die sich im Zuge des "Zeitalters der Konfessionalisierung" jedoch nicht weiter durchsetzen konnte, und es gründet auf den theologischen Forschungen des Ökumenischen Arbeitskreises evangelischer und katholischer Theologen "Lehrverurteilungen - kirchentrennend?" und vor

$6 \quad$ W. Kasper, Martin Luther..., 56.

7 K. Lehmann - W. Pannenberg (dir.), Lehrverurteilungen - kirchentrennend?, Bd. 1: Rechtfertigung, Sakramente und Amt im Zeitalter der Reformation und beute (Herder, Freiburg i. Br./Vandenhoeck \& Ruprecht, Göttingen 1988); K. LeHManN W. Pannenberg (dir.), Lehrverurteilungen - kirchentrennend?, Bd. 2: Materialien zu den Lehrverurteilungen und zur Theologie der Rechtfertigung (Herder, Freiburg i. Br./ Vandenhoeck \& Ruprecht, Göttingen 1989); K. Lehmann - W. PAnnenberg (dir.), Lehrverurteilungen - kirchentrennend?, Bd. 3: Materialien zur Lehre von den Sakramenten und vom kirchlichen Amt (Herder, Freiburg i. Br./Vandenhoeck \& Ruprecht, Göttingen 
allem der 1999 von Vertretern des Lutherischen Weltbundes und der Römisch-katholischen Kirche unterzeichneten Gemeinsamen Erklärung zur Rechtfertigungslehre ${ }^{8}$. Hier ist die Tiefendimension christlichen Glaubens - das Ereignis der Rechtfertigung in Jesus Christus, das, wofür Leben, Tod und Auferstehung Jesu Christi stehen, die Menschwerdung Gottes zum Heil, zur Erlösung und Befreiung von Mensch und Welt als der "gemeinsame" Grund erschlossen, im Blick auf den ein grundlegender Konsens zwischen den christlichen Kirchen formuliert worden ist. Gerade darum ist es entscheidend, das Ereignis der Reformation im Jahr 1517 gemeinsam zu erinnern, und dabei die im Zuge des spannungsreichen, in viele Konflikt- und Gewaltgeschichten eingebetteten Prozesses der geschichtlichen Entwicklung vergessenen, marginalisierten oder unterdrückten Facetten der Reformation und Reformbewegungen der frühen Neuzeit offenzulegen?.

In den folgenden Überlegungen werden zentrale Aussagen der beiden für das Reformationsgedenken wichtigen Dokumente "Vom Konflikt zur Gemeinschaft" und "Erinnerung heilen - Jesus Christus bezeugen" vorgestellt und anschließend ein hermeneutischer Rahmen für das geforderte internationale und ökumenische Reformationsgedenken skizziert, der im interkulturellen Gespräch der Theologien des Nordens mit denen des Südens weiter zu entwickeln ist. Er steht im Kontext der "Kirche des Aufbruchs", zu der der Papst "vom Ende der Welt", Franziskus, entscheidende Impulse gibt, die auch für eine Neubesinnung auf

1990); K. Lehmann - W. Pannenberg (dir.), Lehrverurteilungen - kirchentrennend?, Bd. 4: Antworten auf kirchliche Stellungnahmen (Herder, Freiburg i. Br./Vandenhoeck \& Ruprecht, Göttingen 1994); Die Deutschen BischöFe, Stellungnabme der Deutschen Bischofskonferenz zur Studie Lebrverurteilungen - kirchentrennend?, editado por el Sekretariat der Deutschen Bischofskonferenz (Bonn 1994).

$8 \quad$ Vgl. vor allem: "Gemeinsame Erklärung zur Rechtfertigungslehre des Lutherischen Weltbundes und der Katholischen Kirche", en H. Meyer - D. Papandreou - H. J. URBAN - L. VISCher (dir.), Dokumente wachsender Übereinstimmung: Sämtliche Berichte und Konsenstexte interkonfessioneller Gespräche auf Weltebene, Bd. 3: 1990-2001 (Bonifatius, Paderborn 2003) 419ff; Reformation 1517-2017. Ökumenische Perspektiven: Für den Ökumenischen Arbeitskereis evangelischer und katholischer Theologen, editado por Dorothea Sattler und Volker Leppin (Vandenhoeck \& Ruprecht, Göttingen 2014).

9 Vgl. die für diesen ökumenischen Blick auf Martin Luther wichtigen Studien von: V. LePpin, Die fremde Reformation: Luthers mystische Wurzeln (C. H. Beck, München 2016); V. Leppin, Martin Luther: Vom Mönch zum Feind des Papstes (Lambert Schneider, Darmstadt $\left.{ }^{2} 2015\right)$. 
66 | Margit Eckholt

den Dienst des Papstes für die Einheit der Kirchen und den Frieden in der Welt von entscheidender Relevanz sind.

2. "Vom Konflikt zur Gemeinschaft" und "Erinnerung heilen - Jesus Christus beZeugen": ZWeI KIRCHLiche DoKUMENTE IM Blick auf DAS 500-JÄHRIGE REFORMATIONSGEDENKEN

2.1 "Vom Konflikt zur Gemeinschaft" - das Zentrum der Rechtfertigungsbotschaft im ökumenischen Dialog neu erschließen

Die ökumenischen Perspektiven im Blick auf das Reformationsgedenken, die in den beiden Dokumenten "Vom Konflikt zur Gemeinschaft" und "Erinnerung heilen - Jesus Christus bezeugen" vertreten werden, gründen aus katholischer Sicht in den theologischen und ekklesiologischen Grundlagen für die Ökumene, die das 2. Vatikanische Konzil mit der Kirchenkonstitution "Lumen Gentium" (LG) und dem Ökumenismusdekret "Unitatis Redintegratio" (UR) vorgelegt hat ${ }^{10}$. Hier werden entscheidende Grundlagen für die Achtung vor den anderen und die Anerkennung der anderen christlichen Kirchen gelegt: Das lebendige und Leben schaffende Wort Gottes kann auch bei den vielen anderen entdeckt werden, und sogar noch mehr: Bei den anderen kann gerade auch das entdeckt werden, was in der Tradition der eigenen Kirche vergessen oder in den Hintergrund geraten ist. Diese Anerkennung gründet in der Freiheit und Liebe, und mit ihr ist eine je neue Bekehrung hin zum Wort des Evangeliums verbunden.

"Man darf auch nicht übersehen, dass all das, was durch die Gnade des Heiligen Geistes bei den getrennten Brüdern bewirkt wird, auch zu unserer Erbauung beitragen kann. Alles, was wahrhaft christlich ist, ist niemals den echten Gütern des Glaubens entgegengesetzt, vielmehr kann es immer bewirken, dass das Mysterium Christi und der Kirche selbst vollkommener erreicht wird" (UR 4).

10 Vgl. "Dogmatische Konstitution über die Kirche «Lumen gentium»", en Herders theologischer Kommentar zum Zweiten Vatikanischen Konzil, Bd. 1, editado por P. Hünermann y B.J. Hilberath (Herder, Freiburg i.Br. 2004) 73-185; "Dekret über den Ökumenismus «Unitatis redintegratio»", en Herders theologischer Kommentar zum Zweiten Vatikanischen Konzil, Bd. 1, editado por P. HünERMann (Herder, Freiburg i.Br. 2004) 211-241; W. THÖNISSEN (dir.), „Unitatis redintegratio“: 40 Jabre Ökumenismusdekret - Erbe und Auftrag (Bonifatius, Paderborn 2005). 
Gerade darum ist auch um Verzeihung zu bitten, und so gehören im Blick auf das Gedenken der Reformation öffentliche Bußakte im Rahmen von liturgischen Feiern zu den Konkretionen dieses Gedankens - das ist die zentrale Leitperspektive des Reformationsgedenkens der evangelischen und katholischen Kirche in Deutschland, wie sie im Dokument "Erinnerung heilen - Jesus Christus bezeugen" entfaltet wird.

Diese neuen ökumenischen Perspektiven und das Verhältnis der katholischen Kirche zu anderen christlichen Kirchen waren in den letzten Jahren nicht unbestritten. Das Dokument "Dominus Jesus" $(2000)^{11}$ hat angesichts seiner katholisch-exklusivistischen ekklesiologischen Position gerade unter evangelischen Christen und Christinnen große Bestürzung hervorgerufen, die Ökumene ist belastet worden. Dies ist auch im Schreiben der Glaubenskongregation vom 29. Juni 2007 "zu einigen Aspekten bezüglich der Lehre über die Kirche" aufgegriffen worden. Die Kirchen der Reformation, so wird dort formuliert, "können nach katholischer Lehre nicht «Kirchen» im eigentlichen Sinn genannt werden"12. Auch unter jüngeren katholischen Theologen und Theologinnen (so z.B. Andrea von Teuffenbach ${ }^{13}$ ) nehmen die Interpretationen des „subsistit“ zu, die diese weite Formulierung im Sinne einer Identitätslogik interpretieren und mit einem „est" gleichsetzen. Die eine und einzige Kirche, in der der christliche Glaube als die eine Wahrheit der Welt vermittelt wird, ist die katholische Kirche. Einige haben darum nach "Dominus Jesus" - nach guten Jahren einer Zielperspektive der Ökumene im Sinne der "Einheit in Verschiedenheit" und der Erarbeitung eines "differenzierten Konsenses" in Lehrfragen - von einer "Rückkehrökumene" gesprochen, das heißt: ökumenische Arbeit ist gewünscht, aber im Sinne einer Rückkehr der anderen christlichen Kirchen in den Schoß der katholischen Kirche. ${ }^{14}$ Damit werden die her-

11 M. J. Rainer, „Dominus Jesus“: Anstößige Wahrheit oder anstößige Kirche? Dokumente, Hintergründe, Standpunkte und Folgerungen (LIT, Münster 2001).

12 Kongregation für die Glaubenslehre, Antworten auf Fragen zu einigen Aspekten bezüglich der Lehre über die Kirche, 29. Juli 2007, en http:// www.vatican.va/roman_curia/congregations / cfaith/documents / rc_con_cfaith_doc_20070629_responsa-quaestiones_ge.html

13 A. von Teuffenbach, Die Bedeutung des subsistit in (LG 8): Zum Selbstverständnis der katholischen Kirche (Utz Wiss., München 2002).

14 Einen Überblick über die ökumenischen Perspektiven nach dem 2. Vatikanischen Konzil hat Kardinal Kurt Koch vorgelegt in: „Ökumene im Wandel. Zum Zukunftspotential des Ökumenismusdekrets Unitatis redintegratio“, en J.-H. TÜCK 
meneutischen Grundlagen eines Dialogs mit den anderen christlichen Kirchen und Gemeinschaften, wie sie das Ökumenismusdekret vorgelegt hat, außer acht gelassen und die Grenzen zu den "Anderen" wieder hochgezogen.

Gerade darum ist es von Bedeutung, dass eine lutherisch-katholische Dialogkommission das Dokument "Vom Konflikt zur Gemeinschaft. Gemeinsames lutherisch-katholisches Reformationsgedenken 2017" als Anstoß zur Vorbereitung des Gedenkens an die Reformation vorgelegt hat. Die internationale lutherisch/römisch-katholische Kommission für die Einheit wurde vom Päpstlichen Rat für die Einheit der Christen (Vatikan) und dem Lutherischen Weltbund (Genf) eingesetzt, Generalsekretär Martin Junge und Kurt Kardinal Koch haben das Dokument gemeinsam während der Ratstagung des Lutherischen Weltbundes 2013 in Genf vorgestellt. Das Dokument macht darauf aufmerksam, dass das Gedenken (nicht Jubiläum!) der Reformation in einer globalisierten Welt und in Zeiten der Ökumene erfolgt, was bedeutet, dass ein "gemeinsames Gedenken" notwendig ist und dass auch der Blick der Kirchen des Südens für dieses Gedenken von Bedeutung ist. In Zeiten, in denen es weltweit zu massiven religiösen Pluralisierungsprozessen und gleichzeitig weiteren Säkularisierungsschüben kommt und nicht nur in den Kirchen des Nordens Glaubenstraditionen und Glaubenswissen wegbrechen, in denen für Katholiken oder Protestanten in gleicher Weise kaum (oder nicht) mehr bekannt ist, was die Kirchen getrennt hat, muss das ökumenische Gedenken der Reformation daran erinnern, wofür Martin Luther und seine Theologie gestanden haben und stehen, was Ausgangspunkt der Reformation gewesen ist, welche theologischen Anfragen Luther an die katholische Kirche gestellt hat. Das ist zentraler Inhalt des Dokuments "Vom Konflikt zur Gemeinschaft", aber genau im Sinn eines gemeinsamen Erinnerns an die Reformation; die von Luther und den anderen Reformatoren formulierten Fragen sind heute ein Auftrag für alle christlichen Kirchen, sich mit dem die Kirchen tragenden einen Grund - der Offenbarung Gottes in Jesus Christus und der sich darin für die Menschen ereignenden Erlösung, Rechtfertigung und Befreiung - auseinanderzusetzen und darin der Suche nach der Einheit neue Horizonte zu erschließen. Das Christentum hat sich in verschie-

(dir.), Erinnerung an die Zukunft. Das Zweite Vatikanische Kon₹il (Herder, Freiburg/ Basel/Wien 2012) 335-368. 
denen Konfessionen ausgeprägt, die in komplexen und schwierigen geschichtlichen Prozessen entstanden sind. Den Pluralismus der Konfessionen ernst zu nehmen, ohne dabei die Perspektive des Weges auf eine Einheit hin aufzugeben, beinhaltet dabei die Chance, den Pluralismus innerhalb jeder Konfession zu entdecken. Das wird auch ein spannendes neues Licht auf die Aufgabe werfen, aus jeder Konfession und im Dialog der vielen nach außen und nach innen zu bestimmen, was Einheit, Apostolizität, Heiligkeit und Katholizität der Kirche bedeuten. Genau eine solche plurale und facettenreiche theologische Arbeit an den "notae ecclesiae", den fundamentaltheologischen Grundbestimmungen der Kirche, wird ein entscheidender Beitrag zur Einheit der Kirche sein.

Die Erinnerung an die Reformation vor 500 Jahren muss sich am zentralen Anliegen der Reformation orientieren und dieses in unsere Gegenwart übersetzen. Die Verfasser des Berichtes "Vom Konflikt zur Gemeinschaft" formulieren:

"Die Frage "Wie kriege ich einen gnädigen Gott?» war die Frage, die ihn (d.h. Luther, M.E.) umtrieb. Er fand den gnädigen Gott im Evangelium von Jesus Christus. "Wahre Theologie und Gotteserkenntnis sind im gekreuzigten Christus.» (Heidelberger Disputation) Wenn im Jahr 2017 katholische und evangelische Christen auf die Ereignisse vor 500 Jahren zurückblicken, dann tun sie das am angemessensten, wenn sie dabei das Evangelium von Jesus Christus in den Mittelpunkt stellen. Das Evangelium soll gefeiert und an die Menschen unserer Zeit weitergegeben werden, damit die Welt glaube, dass Gott sich uns Menschen schenkt und uns in die Gemeinschaft mit sich und seiner Kirche ruft. Das ist der Grund für unsere Freude im gemeinsamen Glauben" (Vorwort, S. 10).

Genau das ist die Leitlinie der "Rechtfertigungslehre", "die die Botschaft des Evangeliums zum Ausdruck bringt und deshalb «die gesamte Lehre und Praxis der Kirche unablässig auf Christus hin orientieren will»" (Vorwort, S. 10), wie es die 1999 verabschiedete Gemeinsame Erklärung zur Rechtfertigungslehre ausgedrückt hat.

Der Bericht geht in den zentralen Kapiteln 2, 3 und 4 in knapper, aber differenzierter Weise auf die Entwicklung des Konfliktes um Martin Luther, seine 95 Thesen zur Ablasspraxis und die Debatten um die Theologie Luthers ein, wie sie auf dem Konzil zu Trient (1545-1563) geführt worden sind. Der Bericht macht deutlich, dass die protestantischen Mittelalterforschungen auf der einen Seite und die katholischen 
Lutherforschungen auf der anderen Seite einen wichtigen Beitrag geleistet haben, ideologische Feindbilder zu überwinden, die Reformation in die spätmittelalterlichen Reformbewegungen einzubetten und nicht Luthers Rechtfertigungslehre als trennendes Moment der Bewegung der Reformation zu sehen, sondern die Auseinandersetzungen um die (notwendige) Reform der Kirche. Luther hatte sich an der Ablasspraxis der Kirche gestört und in seinem Schreiben vom 31.10.1517 mit der Überschrift "Disputation zur Klärung der Kraft der Ablässe" in den 95 Thesen den Erzbischof Albrecht von Mainz und seinen Diözesanbischof Hieronymus Schulze von Brandenburg um Klärungen gebeten (Nr. 40, S. 29). "Nach Luthers Urteil", so der Bericht, "fügte die Ablasspraxis der Frömmigkeit der Christen Schaden zu. Er stellte in Frage, ob Ablässe die Büßenden von Strafen, die Gott ihnen auferlegt hatte, befreien könnten; ob Strafen, die ein Priester auferlegt hatte, ins Fegfeuer übertragen werden könnten; ob nicht der heilende und reinigende Aspekt der Strafen bedeutete, dass ein ernsthaft Büßender es vorziehen würde, die Strafen zu erleiden, statt von ihnen befreit zu werden; und ob das Geld, das für Ablässe gegeben wurde, nicht besser den Armen gegeben werden sollte. Er stellte auch die Frage nach dem Wesen des Kirchenschatzes, aus dem der Papst Ablässe anbot" (Nr. 45, S. 29).

Der Disput um den Ablass hat Martin Luther das Zentrum theologischer Gnaden- und Erlösungslehre neu verstehen lassen und zur Formulierung der Rechtfertigungsbotschaft geführt. Die historischen und theologischen Arbeiten der Dialogkommissionen haben in den letzten Jahren dazu beigetragen, dass genau dieses zentrale Anliegen der Reformatoren nicht mehr Anlass für Ausgrenzungen sein kann ${ }^{15}$. Auch durch Vorbereitungen in den USA, so die Veröffentlichung des Dokuments "Rechtfertigung durch Glauben", wurde 1999 die Gemeinsame Erklärung zur Rechtfertigung von Vertretern des Lutherischen Weltbundes und des römischen Einheitssekretariats unterzeichnet, die einen Konsens in den grundlegenden Wahrheiten der Rechtfertigungslehre formuliert (Nr. 25, S. 22). Das ist ein entscheidendes Moment, um die Geschichte der Reformation heute "gemeinsam erzählen" zu können (Nr. 35, S. 27). Der Bericht der Dialogkommission fasst das Recht-

15 Vgl. Lutherisch/Römisch-Katholischer Dialog in den USA, „Rechtfertigung durch den Glauben (1983)", en Rechtfertigung im ökumenischen Dialog: Dokumente und Einführung, editado por H. MeYer - G. Gassmann (Lembeck, Frankfurt a. M. 1987) 107-200; Gemeinsame Erklärung zur Rechtfertigungslebre. .., 419 ff. 
fertigungsgeschehen "sola gratia" - "solus Christus" folgendermaßen zusammen:

"Das Bild (des «fröhlichen Wechsels», d.h. dass Jesus Christus in mir lebt, M.E.) zeigt, dass etwas Äußeres, nämlich die Gerechtigkeit Christi, zu etwas Innerem wird. Sie wird Eigentum der Seele, aber nur in der Gemeinschaft mit Christus durch das Vertrauen in seine Verheißungen, nicht unabhängig von ihm. Luther besteht darauf, dass unsere Gerechtigkeit etwas ganz Äußeres ist, weil sie Christi Gerechtigkeit ist, aber sie muss zu etwas gänzlich Innerem werden durch den Glauben an Christus. Nur wenn beide Seiten in gleichem $\mathrm{Maß}$ betont werden, wird die Wirklichkeit des Heils zureichend verstanden. Luther stellt fest: «Im Glauben selbst ist Christus gegenwärtig». Christus ist "für uns» (pro nobis) und in uns (in nobis), und wir sind in Christus (in Christo)" (S. 50, Nr. 108).

Im Blick auf die kontroverstheologische Auseinandersetzung um Luthers Verhältnis zum Gesetz formuliert der Bericht:

"Luther hat andererseits die Wirklichkeit des Menschen mit Bezug auf das Gesetz in seiner theologischen oder geistlichen Bedeutung wahrgenommen, also aus der Perspektive dessen, was Gott von uns fordert. Jesus bringt den Willen Gottes zum Ausdruck, wenn er sagt: «Du sollst den Herrn, deinen Gott, lieben von ganzem Herzen und mit deiner ganzen Seele und mit deinem ganzen Verstand» (Matthäus 22,37). Das bedeutet, dass die Gebote Gottes nur erfüllt werden in einer völligen Hingabe an Gott. Das schließt nicht nur den Willen und die entsprechenden äußeren Handlungen ein, sondern alle Aspekte der menschlichen Seele und des menschlichen Herzens wie Gefühle, Verlangen, Streben, also auch jene Aspekte der Seele, die entweder nicht unter der Kontrolle des Willens sind oder nur indirekt und teilweise durch die Tugenden" (S. 51, Nr. 109).

Dieser Zugang zur Tiefendimension christlichen Glaubens wird von den beiden christlichen Konfessionen bekannt: "Gemeinsam bekennen Katholiken und Lutheraner: "Allein aus Gnade im Glauben an die Heilstat Christi, nicht auf Grund unseres Verdienstes werden wir von Gott angenommen und empfangen den Heiligen Geist, der unsere Herzen erneuert und uns befähigt und aufruft zu guten Werken»(GE 15). Der Ausdruck «allein aus Gnade» wird folgendermaßen weiter erklärt: «die Botschaft von der Rechtfertigung [.. . ] sagt uns, dass wir Sünder unser neues Leben allein der vergebenden und neuschaffenden Barmherzigkeit Gottes verdanken, die wir uns nur schenken lassen und im Glau- 
ben empfangen, aber nie - in welcher Form auch immer - verdienen können» (GE 17)" (S. 55, Nr. 124). Dann werden die theologischen Differenzpunkte - das Verhältnis von Gnade und Freiheit - benannt und aus lutherischer und katholischer Perspektive beleuchtet, um das Gemeinsame herauszuarbeiten:

"Weil Glaube nicht nur als mit Zustimmung verbundenes Wissen verstanden wird, sondern als Vertrauen des Herzens, das sich auf das Wort Gottes gründet, kann weiter gemeinsam gesagt werden: «Rechtfertigung geschieht sallein durch Gnade» (GE 15 und 16), allein durch Glauben, der Mensch wird «unabhängig von Werken gerechtfertigt (Röm 3,28; vgl. GE 25)» (GE, Annex 2C)” (S. 56, Nr. 128).

Das ist darin begründet - und hier knüpft der Bericht im Grunde an die klassischen Axiome der Theologie von Thomas von Aquin an -, dass Christen "im eigentlichen und strengen Sinn" "nicht an die Rechtfertigung und an die Kirche" glauben, "sondern an den Vater, der sich unser erbarmt und uns in der Kirche als sein Volk versammelt, an Christus, der uns rechtfertigt und dessen Leib die Kirche ist, und an den Heiligen Geist, der uns heiligt und der in der Kirche lebt. Unser Glaube erstreckt sich auf Rechtfertigung und Kirche als Werke des dreifaltigen Gottes, die nur im Glauben an ihn richtig angenommen werden können"(S. 88, Nr. 217). Hier bezieht sich das Dokument "Vom Konflikt zur Gemeinschaft" auf den Bericht der Gemeinsamen Römisch-katholischen/Evangelisch-lutherischen Kommission "Kirche und Rechtfertigung. Das Verständnis der Kirche im Licht der Rechtfertigungslehre" (1993, Nr. 5) und knüpft auch an die darin formulierten Desiderate $a^{16}$.

Das Dokument vertritt die Position einer "Konsensökumene" und knüpft damit an die Fortschritte in der Ökumene in der Nachkonzilszeit an. Es hat sich in den "lutherisch-katholischen Gesprächen" "ein klarer Konsens herausgebildet, dass die Lehre von der Rechtfertigung und die Lehre von der Kirche zusammengehören” (Nr. 216, S. 87). Sicher sind auf Zukunft hin weitere ökumenische Gespräche notwendig: über die Beziehung zwischen Sichtbarkeit und Unsichtbarkeit der

16 Vgl. auch: Rechtfertigung und Freibeit: 500 Jahre Reformation 2017: Ein Grundlagentext des Rates der Evangelischen Kirche in Deutschland (EKD) (Gütersloher Verlagshaus, Gütersloh ${ }^{4}$ 2015); Rechtfertigung heute - Warum die zentrale Einsicht Martin Luthers zeitlos aktuell ist, editado por el Amt der VELKD (Lutherisches Kirchenamt, Hannover 2016). 
Kirche, die Beziehung zwischen Universalkirche und Ortskirche, über die Kirche als Sakrament, über die Notwendigkeit der sakramentalen Ordination im Leben der Kirche und über den sakramentalen Charakter der Bischofsweihe (vgl. Nr. 218, S. 88). Das ist gerade darum von Bedeutung, weil „Katholiken und Lutheraner nie aufgehört haben, miteinander den Glauben an die «eine, heilige, katholische und apostolische Kirche» zu bekennen" (Nr. 218, S. 87).

2.2 "Erinnerung heilen - Jesus Christus bezeugen": in gemeinsamer heilender Erinnerung ein Christusfest feiern

Die beiden letzten Kapitel des Berichtes "Vom Konflikt zur Gemeinschaft" formulieren Konkretionen im Blick auf die Vorbereitung und Durchführung des Gedenkens an die Reformation, die dann von den evangelischen und katholischen Bischöfen in Deutschland in ihrem Dokument "Erinnerung heilen - Jesus Christus bezeugen" aufgegriffen worden sind. Gemeinsam der 500 Jahre der Reformation zu gedenken, bedeutet Verbindendes und Trennendes zu erinnern. "Dieses Ringen hat zwei Seiten: die Anerkennung dessen, was ihnen gemeinsam ist und sie miteinander verbindet, und die Anerkennung dessen, was sie trennt. Das Erste ist Anlass zu Dankbarkeit und Freude; das Zweite ist Anlass für Schmerz und Klage" (Vom Konflikt zur Gemeinschaft, Nr. 223, S. 90). Dazu gehört auf der einen Seite, ein "Christusfest" zu feiern, gerade weil die Reformatoren in ihrem Zugang zur Schrift Jesus Christus in das Zentrum gestellt haben und weil die Taufe verbindet ${ }^{17}$. Auf der anderen Seite gilt es, gemeinsam die Schuld zu bekennen, die sowohl auf katholischer als auch protestantischer Seite begangen worden ist. Die deutsche katholische Bischofskonferenz (DBK) und die Evangelische Kirche in Deutschland (EKD) haben für den zweiten Fastensonntag $2017 \mathrm{zu}$ einem solchen Bußgottesdienst nach Hildesheim eingeladen; auch auf Ebene der verschiedenen Diözesen in Deutschland und auf Ebene von Pfarreien werden ähnliche Feiern vorbereitet.

„Das Zweite Vatikanische Konzil lehrt, dass Menschen, die getauft sind und an Christus glauben, jedoch nicht der Römisch-katholischen Kirche angehören, «durch den Glauben in der Taufe gerechtfertigt und dem Leibe Christi eingegliedert (sind)»; «mit Recht werden sie von den Söhnen der katholischen Kirche als Brüder im Herrn anerkannt» (UR 3). Evangelische Christen sagen dasselbe über ihre katholischen Mitchristen“ (Vom Konflikt zur Gemeinschaft, Nr. 220, S. 88). 
Das Dokument "Erinnerung heilen - Jesus Christus bezeugen" legt den Entwurf eines ökumenischen Bußgottesdienstes vor; die theologischen Grundlagen dazu werden in den fünf Kapiteln des Dokuments gelegt. Von Bedeutung ist die Erarbeitung des Konzeptes einer "heilenden Erinnerung", das in einer zweifachen Perspektive erschlossen wird: als "Heilung der Erinnerungen" und als "Heilung durch Erinnerung". Damit das Reformationsgedenken 2017 zu einem ökumenischen Ereignis wird, ist es notwendig, Verletzungen auszusöhnen und einen Prozess des gemeinsamen Heilwerdens anzustoßen. Mit dem Stichwort "healing of memories" wird angeknüpft an die Versöhnungsprozesse in politischen Kontexten von Gewalt und Frieden, so in Südafrika oder in anderen afrikanischen Ländern und in den Balkanländern, wo unter diesem Titel ein komplexer Prozess der Aussöhnung der verschiedenen Völker angestoßen worden ist. Heilung der Erinnerung und Heilung durch Erinnerung gehören dabei zusammen: Der Heilungsprozess

"kann dazu führen, dass sich Wunden schließen und Narben nicht mehr schmerzen. Betont man hingegen stärker das Moment, dass die bewusst gemachten Erinnerungen selbst eine heilsame Kraft entfalten können, dann rückt die Heilung durch die Erinnerungen in den Blick. Das geschieht immer dann, wenn man sich erinnernd in die Lage der jeweils anderen hineinzuversetzen vermag und die Geschichten, die zwischen ihnen und uns stehen, aus ihrem Blickwinkel zu sehen lernt. Die Heilung der Erinnerungen und die Heilung durch Erinnerungen bedingen sich gegenseitig. Am Ende stehen auf jeden Fall ein vertieftes Verständnis füreinander, wechselseitige Empathie und schließlich Aussöhnung"18.

Wichtig ist, sich die "Orte" der Reformation und des Bruchs der einen westlichen Kirche zu vergegenwärtigen und die theologischen Debatten - hier werden wie im Dokument "Vom Konflikt zur Gemeinschaft" die Rechtfertigungslehre, Fragen der Kirchengemeinschaft, der Abendmahls- und Eucharistiegemeinschaft genannt - gemeinsam neu zu erschließen. Für die katholische Kirche wird es darum gehen anzuerkennen, dass Luther die Kirche unter den "befreienden Ruf des Evangeliums" stellen wollte und "aus dem Geist des Evangeliums umfassend erneuern wollte" ${ }^{19}$. Für die evangelische Kirche wird es wichtig sein einzusehen, dass es der katholischen Kirche in gleicher Weise um Er-

\footnotetext{
Erinnerung beilen ..., 16.

19 Erinnerung beilen..., 60.
} 
neuerung ging in der Gemeinschaft mit dem Papst und den Bischöfen. Heute sei es wichtig, sich im ökumenischen Gespräch mit den "Erinnerungsorten" auseinanderzusetzen, d.h. den Orten, die verletzt haben und an denen sich in Zeiten der Konfessionalisierung Konfliktlinien kristallisiert haben. Das Dokument nennt hier: die Gestalt Luthers, den Reichstag in Worms 1521, den Bauernkrieg und den 30-jährigen Krieg, die Konfessionskarte in Deutschland, die bis heute von einer der zentralen Differenzen geprägt ist, die sich an der Auseinandersetzung Luthers mit dem Papst festmacht: Das ist auf der einen Seite die Erinnerung an den Bann über Luther, am 3.1.1521 vom Papst ausgesprochen, und auf der anderen Seite ist es die Verwerfung des Papsttums durch Luther, wie er sie in seiner Schrift "De captivitate babylonica" formuliert hat ${ }^{20}$. Gleichzeitig wird aber das herausgestellt, was für Luther und die katholische Kirche damals und heute von zentraler Relevanz ist und was heute gemeinsam in einem veränderten gesellschaftlichen und kulturellen Kontext zu erschließen ist: die Buße, die Erfahrung des Menschen, vor Gott, den anderen und sich selbst immer wieder zu versagen und doch aus der Tiefe des heilenden Vor-Gott-Stehens je neu Erlösung und Heil zu erfahren.

"Luther hat mit den Ablassthesen, die er 1517 veröffentlicht, die Buße ins Zentrum gestellt und daran immer festgehalten: Menschen können und sollen ihre Sünden im Vertrauen auf Gottes Gnade bekennen; sie empfangen die Vergebung durch Jesus Christus. Der Ruf zur Umkehr hat nichts an Aktualität verloren. Der reformatorische Grundimpuls zielt auf die Umkehr der Kirche zu Jesus Christus. So sehen wir uns als Kirchen im 21. Jahrhundert zur Reform und Erneuerung gerufen; die Heilung der Erinnerung gehört in diesen Zusammenhang. Wir wollen durch unsere ökumenische Initiative zeigen, dass dem Glauben an Gott nichts Zerstörerisches innewohnt, sondern dass er dem Frieden dient, weil er Schuld, Not und Leid, Hass und Feindschaft überwindet. Dafür stehen wir ein und lassen uns daran messen, dass die Verantwortung vor Gott die Freiheit der Menschen nichtbeeinträchtigt, sondern fördert. Wir können in der Auseinandersetzung mit unserer eigenen Geschichte zeigen, dass Vielfalt und Einheit keine Gegensätze sein müssen, sondern einander bedingen, wenn das menschliche Miteinander vom Geist Gottes inspiriert ist und sich von Jesus Christus leiten lässt"21.

Erinnerung heilen..., 34-36.

21 Erinnerung beilen ..., 64. 
Das Dokument ruft zu einer Auseinandersetzung mit den theologischen Fragen in genau dem Sinne, "das Gemeinsame zu suchen" und formuliert einen wichtigen hermeneutischen Grundsatz:

"Um das rechte Verständnis der Wahrheit des Evangeliums muss weiter gerungen werden, aber das kann nicht so geschehen, dass sich die einen auf Kosten der anderen profilieren. Die Heilung der Erinnerungen ist ein Weg, der hier neue Horizonte erschließt. Wer daran teilnimmt, verpflichtet sich, die eigene Geschichte mit den Augen des anderen zu betrachten und sich in die Geschichte des anderen zu versetzen. Auf diese Weise entstehen Freiheit für Schuldeinsicht und Empathie. Die Heilung der Erinnerungen ist eingebettet in die Geschichte des ökumenischen Lernens und nimmt die wechselseitigen produktiven Erfahrungen in der Diakonie und Caritas auf. Im Kern handelt es sich um einen geistlichen Prozess, der Schuld und Vergebung, die gottesdienstliche Zuwendung zu Gott und die engagierte Liebe zum Nächsten in sich schließt"22.

Auch über das Reformationsgedenken hinaus wird das Dokument von Bedeutung sein. Die "Heilung der Erinnerungen" und die "Heilung durch die Erinnerung" werden die christlichen Kirchen in Zukunft weiter begleiten, und es in ein Prozess, der über den deutschsprachigen Kontext hinaus auch in internationaler Perspektive von Bedeutung sein wird. In einer befreiungstheologischen Perspektive wird er in dem Sinn neu zu formulieren sein, dass das Reformationsgedenken nicht ein innerkirchliches Geschehen ist, sondern bezogen sein muss auf den Dienst an der Einheit der Kirchen und den Dienst an der zusammenwachsenden Menschheit, einem Miteinander in Gerechtigkeit und Frieden und im Dienst eines "guten Lebens" 23 . Das ist dann das Christusfest, von dem die katholischen und evangelischen Bischöfe im Dokument "Erinnerung heilen - Jesus Christus bezeugen" sprechen und was, so Kardinal Walter Kasper, die „beste ökumenische Idee für das Jahr 2017“24 ist: "Die Heilung der Erinnerung befreit uns zu einem gemeinsamen und darin glaubwürdigeren Zeugnis für Jesus Christus. Sie ermutigt uns, das

2 Erinnerung beilen ..., 61.

23 Die Buß- und Versöhnungsgottesdienste weisen über sich hinaus: „Sie stehen in innerer Verbindung sowohl zu caritativen und diakonischen Aktionen als auch zu katechetischen und theologischen Vorhaben, die dem Geist der Ökumene und eines geschwisterlichen Miteinanders entsprechen. So kann die Heilung der Erinnerung zu einer breiten Bewegung werden“" (Erinnerung heilen..., 62).

24 W. Kasper, Martin Luther..., 56. 
bevorstehende Reformationsjubiläum gemeinsam als ein Christusfest zu begehen"25.

3. Reformation und WeltKirChlichen Aufbruch "Miteinander lesen" neue ÖKumenische Perspektiven im Dienst der Einheit Der Kirche und des Friedens in Der Welt

Der französische Philosoph Paul Ricoeur hat in seinen hermeneutischphilosophischen Überlegungen zum Prozess des Erinnerns herausgearbeitet, dass sich Identität nur in und über vielfältige und kontinuierliche Prozesse der Erinnerung konstituiert und dass sie immer in Gestalten der Alterität eingebettet ist; wir sind „in Geschichten verstrickt“, mit den vielen anderen, denen wir begegnen ${ }^{26}$. Die "Entdeckung des bzw. der anderen" ist Teil der Konstitution von Identität, das ist Konsens philosophischer und pädagogischer Theorien der Identität, und ein solches Konzept führt zu einem neuen Blick auf die Geschichte und die sie prägenden Eroberungs-, Ausgrenzungs- und Gewaltgeschichten. Nur im Hören auf die Fremden und im Wahrnehmen der vielen "anderen" auch herausfordernden und befremdenden - Geschichten kann ich die Frage nach meiner "katholischen" christlichen Identität oder andere die nach ihrer "protestantischen" christlichen Identität stellen. Die Antwort ergibt sich aus vielfältigen Begegnungen und in der Übersetzung hin zum anderen, wobei sich, so formuliert es Paul Ricoeur, im Austausch der Erinnerungen einerseits die Schuld der Geschichte und die uneingelösten Versprechen melden, andererseits können aber genau hier, in dem der tiefste Grund der "mémoire" - des Gedächtnisses - aufgedeckt und re-aktiviert wird, die in der "mémoire" liegenden "Versprechen" eine neue, prospektive Kraft entfalten ${ }^{27}$. Diese Weite der Erinnerung ist der gemeinsame Raum, in dem sich das Evangelium mit seinen heilenden und Zukunft eröffnenden Kräften einspielen kann; es ist der Raum, in dem in theologischer Perspektive für die katholische Kirche und die Kirchen der Reformation Ökumene möglich ist, weil sich hier der Zukunftshorizont meldet, der aus der weiten Perspektive des Reiches Gottes erwächst, das Jesus Christus verkündet hat, den Johannes als

25 Erinnerung beilen ..., 64.

26 P. Ricoeur, Soi-même comme un autre (Editions du Seuil, Paris 1990); id., Gedächtnis, Geschichte, Vergessen. Aus dem Französischen von Hans-Dieter Gondek/Heinz Jatho/Markus Sedlaczek (Fink, München 2004).

27 P. Ricoeur, Gedächtnis..., 760. 
Liebe und Wahrheit bezeichnet (Joh 1,16ff), Paulus als Gerechtigkeit und Freiheit (Röm 3,21; 2 Kor 3,17). Wer an Jesus Christus glaubt, wird zu einer "neuen Schöpfung": Er lebt in der Freiheit der Kinder Gottes, und gerade darin wird das Gesetz erfüllt: "Wenn also jemand in Christus ist, dann ist er eine neue Schöpfung: Das Alte ist vergangen, Neues ist geworden. Aber das alles kommt von Gott, der uns durch Christus mit sich versöhnt und uns den Dienst der Versöhnung aufgetragen hat" (2 Kor $5,17 f$.$) .$

Die beiden Dokumente "Vom Konflikt zur Gemeinschaft" und "Erinnerung heilen - Jesus Christus bezeugen" leiten an, im Kontext des 500jährigen Gedenkens der Reformation den gemeinsamen und internationalen Horizont dieses Gedenkens zu erschließen. Das bedeutet dann auch, wird das mit Paul Ricoeur benannte weite Geschichtsverständnis vorausgesetzt, den Blick auf die Zeit der Reformation in ökumenischer Perspektive zu weiten. Aus katholischer Perspektive war die Zeit der Reformation auch die Zeit der "Entdeckungen" und "Eroberungen": 1492 landete Kolumbus auf Hispaniola (die heutige dominikanische Republik), 1487/88 umsegelte Bartolomeu Diaz die Spitze Südafrikas, 1497/98 stieß Vasco da Gama über dieses "Kap der Guten Hoffnung" auf dem Seeweg nach Indien, 1541 bis 1552 verkündete Franz Xaver (1506-1552), einer der ersten Gefährten des Gründers des Jesuitenordens, Ignatius von Loyola, das Evangelium in Asien, er errichtete in Goa die erste Niederlassung der Jesuiten, ein Kolleg zur Ausbildung einheimischer Jugendlicher. In seinen Überlegungen zur spanischen Conquista, die im Kontext des 500-Jahr-Gedenkens der Eroberung und Entdeckung des amerikanischen Kontinents breit diskutiert worden sind, hat der französische Sprachphilosoph und Historiker Tzvetan Todorov den vielschichtigen Prozess der „Entdeckung der anderen“ herausgearbeitet, das Leid, das den eingeborenen Völkern widerfahren ist und zur Etablierung eines europäischen Kolonialsystems geführt, das weit bis in unsere Zeit nachwirkt und die gegenwärtigen indigenen Bewegungen und ihren Widerstand gegen ein globales kapitalistisches Weltsystem prägt; postkoloniale Theoriebildungen, die mittlerweile auch in den Kulturwissenschaften im deutschsprachigen Raum angekommen sind, knüpfen genau daran $\mathrm{an}^{28}$.

28 T. Todonov, Die Eroberung Amerikas: Das Problem des Anderen, Aus dem Französischen von Wilfried Böhringer (Suhrkamp, Frankfurt a.M. 1985); vgl. zu Hermeneutiken der Anerkennung: J. B. Metz, Für eine neue hermeneutische Kultur: Zu Ehren von Edward Schillebeeckx, en Orientierung 53 (1989) 256-259; id., 
Dieses "encubrimiento" („Verdeckung") der neuen Welten ist aber auch in den Jahrzehnten der Entdeckungen und Eroberungen nicht unbestritten geblieben. Mit den beeindruckenden Predigten des Dominikaners Antonio de Montesinos (1475-1545) im Advent 1511 auf Hispaniola und dem Bekehrungsprozess, den sein Mitbruder Bartolomé de las Casas (14841566) vom Encomendero zum Verteidiger der Menschenwürde der Indígenas durchlaufen hat, haben sich Reformbewegungen gemeldet, die sich wie die Reformation aus dem befreienden Geist des Evangeliums gespeist haben. Die Reformation Luthers und die Entdeckungs- und Eroberungsprozesse der "neuen Welten" und die damit von der katholischen Kirche - vor allem von Ordensgemeinschaften wie den Dominikanern, Franziskanern und Jesuiten - getragenen Missionsbestrebungen sind parallel verlaufen, ohne jedoch voneinander beeinflusst zu werden. Es wird heute wichtig sein, gerade aus Perspektive der Theologien des Südens die Dynamik beider Bewegungen "zusammen zu lesen"; genau das wird helfen, die Reformation Luthers "anders zu erzählen"29.

In kirchenhistorischen Darstellungen wird der weltkirchliche Aufbruch im Katholizismus der Frühen Neuzeit mit einem starken gegenreformatorischen Akzent verknüpft, es ist von der "Kompensationstheorie"30 die Rede: Durch die Eroberung der indigenen Völker Lateinamerikas und die damit übereingehende Missionierung der "neuen Welt" wird der territoriale Verlust und der Verlust von Gläubigen wettgemacht. Die Reformation Luthers war demgegenüber nicht mit einer Perspektive der Mission verknüpft, auch bedingt durch die Entstehung von National- und Territorialkirchen und den Konfessionalisierungs-

„Im Eingedenken fremden Leids: Zu einer Brückenkategorie zwischen Theologie und Ethik, zwischen Religion und Moral“", en Katechetische Blätter 122 (1997) 78-87.

Das ist Zielstellung einer Tagung, die vom 30. Juni bis 2. Juli 2017 in Magdeburg zum Thema „Die Zeit der Reformation aus anderem Blickwinkel: Eine lateinamerikanisch-ökumenische Perspektive" von Prof. Dr. Margit Eckholt (Universität Osnabrück) und Prof. Dr. Johannes Meier (Universität Mainz) in Kooperation mit ICALA - Intercambio cultural alemán-latinoamericano durchgeführt wird.

30 Vgl. dazu J. MeIer (dir.), Sendung - Eroberung - Begegnung: Franz Xaver, die Gesellschaft Jesu und die katholische Weltkirche im Zeitalter des Barock (Harrassowitz, Wiebaden 2005); H. J. PRIEN, Die Geschichte des Christentums in Lateinamerika (Vandenhoeck \& Ruprecht, Göttingen 1978), 64: Prien zitiert Júlio Maria und die These, nach der „die Entdeckung Amerikas eine Kompensation für die Kirche in der Epoche war, in der der Protestantismus dem katholischen Glauben die Hälfte Europas entriß““. 
prozess. ${ }^{31}$ Beide Entwicklungen sind jedoch näher miteinander verknüp$\mathrm{ft}$ als bislang gedacht. Wissenschaftliche Ansätze der Gegenwart sowohl in Geschichte, Kirchengeschichte und systematischer Theologie - beziehen Thesen der Globalisierung ein und arbeiten transkulturelle und interkulturelle Verknüpfungen heraus, und auf dem Hintergrund dieser Ansätze, die auch auf befreiungstheologische Hermeneutiken zurückgreifen, die ökumenisch ausgerichtet sind, kann es zu einer neuen Lektüre der Geschichte kommen und werden so im "Gedächtnis" der Reformation ausgeblendete oder verloren gegangene Traditionsspuren aufgedeckt.

Die Reformation gemeinsam erinnern und anders erzählen, wie es das Dokument "Vom Konflikt zur Gemeinschaft" einfordert, wird darum mit diesem neuen - weiten - Blick auf die Geschichte zu tun haben und einem "Heilen der Erinnerung", wie es die evangelischen und katholischen Bischöfe in Deutschland gefordert haben. Das wird für eine Erinnerung in dogmatisch-theologischer Perspektive die Konsequenz haben, die Grundfragen reformatorischer Theologie - die Bedeutung der Rechtfertigung des Menschen durch Jesus Christus und die Rückbindung an das Wort des Evangeliums, wie es in den Schrift-

31 Vgl. dazu die Darstellung in der evangelischen Missionsgeschichte: MarTiN Schlunk, Die Weltmission der Kirche Christi. Ein Gang durch neunzehn Jahrbunderte (Evang. Missionsverlag, Stuttgart ${ }^{2} 1951$ ), 106-108: „Das muß man sich gegenwärtig halten, um das Überraschende des Satzes zu verstehen, man könne eine Geschichte der Reformation schreiben, ohne das Wort Mission zu gebrauchen. Es ist Tatsache, so unbegreiflich es im ersten Augenblick aussieht, daß unsere Reformatoren in Deutschland und der Schweiz so außer Zusammenhang mit dem starken kolonialund Missionsleben ihrer Tage standen, daß sie an Mission im Sinne unserer heutigen Heidenmission nicht gedacht haben. Auch Luther nicht, dessen Stellung zum Missionsgedanken allerdings viel positiver war, als man früher annahm. Obwohl in einer kleinen Kontinentalstadt lebend, hat Luther von den großen Entdeckungen seines Zeitalters gewußt, und es war ihm bei seinem dynamischen Verständnis von Evangelium und Kirche selbstverständlich, daß auf jenen Inseln und Ländern die Botschaft des Evangeliums noch nicht ausgerichtet sei... Zu einer Missionstat ist es bei ihm nicht gekommen. Das wäre auch ganz undenkbar gewesen. Solange der Grundsatz, daß der Landesherr den Glauben der Untertanen bestimme, herrschte und nur katholische Fürsten und Staaten Kolonialbesitz hatten und Kolonialpolitik trieben, war evangelische Heidenmission praktisch unmöglich, ganz abgesehen noch von der Frage, woher die aus dem Zusammenbruch der Mönchs- und Priesterkirche langsam erstehende Kirche der Reformation hätte Menschen und Mittel zur Heidenmission nehmen sollen.“ 
texten bezeugt ist, die "Freiheit des Christenmenschen" und die Bedeutung, die dem "gemeinsamen Priestertum" aller Gläubigen zukommt, die Anfragen an kirchliche, amts- bzw. sakramententheologische Vermittlungsgestalten - in ökumenischer Perspektive unter Berücksichtigung des Welthorizontes, der Herausforderungen der globalen Weltgesellschaft durch vielfältige Konflikte, durch zunehmende Gewalt und auch religiöse Ausschlussprozesse zu erschließen. Eine interkulturelle systematische Theologie, eine Missionswissenschaft und eine Kirchengeschichte in globaler Perspektive, die die Entwicklungen im außereuropäischen Christentum ernst nehmen und sich durch sie "hinterfragen" lassen, werden darauf hinweisen können, wie eng die reformatorische Bewegung im Europa des 16. Jahrhunderts und die gleichzeitige weltweite Expansion der katholischen Kirche doch aufeinander bezogen sind. Die gegenwärtige ökumenische Landschaft ist durch diese beiden Bewegungen gekennzeichnet ist: die konfessionelle Vielfalt als Folge der reformatorischen Bewegung auf der einen Seite, ihre kontextuelle Vielgestaltigkeit, zwangsläufiges Resultat der Ausbreitung des Christentums in unterschiedliche geographische Regionen und Kulturräume auf der anderen Seite. Beide Vorgänge setzten im 15./16. Jahrhundert ein und sind zum Verständnis der aktuellen Ökumene unerlässlich. Die ökumenische Bewegung ist Anfang des 20. Jahrhunderts entstanden; innerprotestantisch hat sie mit der Missionsbewegung zu tun, die im 19. Jahrhundert mit den kolonialen Bestrebungen der europäischen Mächte verknüpft ist, innerkatholisch mit einer Auseinandersetzung mit der Katholizität und der Entwicklung neuer ekklesiologischer Ansätze, die die "societas-perfecta-Ekklesiologie" der frühen Neuzeit aufzubrechen beginnen, sich an den biblischen und patristischen Bildern der Kirche als Volk Gottes und Leib Christi orientieren und den Weg einer Kirchenreform bereiten, für den das 2. Vatikanische Konzil der entscheidende Wendepunkt ist.

Die Reformation "anders erzählen": das ist lebendige Praxis in den ökumenischen Netzwerken, von Frauen und Männern, die mittlerweile seit über 100 Jahren unterwegs sind, auf Ebene von Institutionen (wie dem Weltrat der Kirchen) und theologischen Dialogforen (wie z.B. dem Ökumenischen Arbeitskreis evangelischer und katholischer Theologen oder ökumenisch ausgerichteten Zeitschriften wie "Una Sancta"), "an der Basis" (in der Arbeitsgemeinschaft christlicher Kirchen, aber auch in ökumenischen Arbeitskreisen von Pfarrgemeinden) und im Zuge der 
Entstehung neuer Formen des Kirche-Seins in den Ländern des Südens auch im Zusammenhang befreiungstheologischer Zusammenschlüsse wie EATWOT, der ökumenischen Vereinigung der Theologen und Theologinnen der Dritten Welt. Die befreiungstheologischen Perspektiven, wie sie seit dem 2. Vatikanischen Konzil entfaltet worden sind, wach zu halten und weiter zu schreiben, tut not in einer Welt, die von einer zunehmenden "Fundamentalisierung" von Religion geprägt ist. Auch Christen und Christinnen machen die Erfahrung, dass konfessionelle Grenzen wieder gezogen werden; aus katholischer Perspektive hat das im Jahr 2000 vom damaligen Präfekten der Glaubenskongregation Kardinal Joseph Ratzinger veröffentlichte Dokument "Dominus Jesus" „über die Einzigkeit und die Heilsuniversalität Jesu Christi und der Kirche" dazu beigetragen und die vielfältigen Prozesse der "Entdeckung der anderen” gebremst - nicht nur im Blick auf die Ökumene, ebenso im Blick auf den Dialog der Religionen und die Ausbildung neuer kontextueller und inkulturierter Gestalten von Theologie. Die gegenwärtigen Zeiten sind aber keine Zeiten von Grenzziehungen, diesen Spiegel halten die gerade in den Ländern des Südens rasch wachsenden Pfingstkirchen den Kirchen der Reformation und der katholischen Kirche vor. Mittlerweile sollen weltweit ca. 600 Millionen Menschen der Pfingstbewegung zugehören, im traditionell katholischen Lateinamerika sind es in vielen Ländern schon über 20 Prozent der Bevölkerung. Der "Pentekostalismus" ist ein grenzüberschreitendes Phänomen, eine neue "Grundgestalt" des Christentums, die zur Ausbildung eines neuen „Stils" christlichen Glaubens beiträgt ${ }^{32}$. Die Gefahr einer "Fundamentalisierung” des Glaubens ist dann gegeben, wenn Grenzen gezogen, Menschen ausgeschlossen, wenn Glaubenswahrheiten anderen

32 Vgl. dazu: M. Eckholt, "Eine neue «charismatische Dimension» der Evangelisierung?”, en Zeitschrift für Missionswissenschaft und Religionswissenschaft 98 (2014) 76-90; M. ECKHOLT, "Pfingstkirchen als Herausforderung für den Katholizismus", en MD - Materialdienst des Konfessionskundlichen Instituts Bensheim 65 (2014) 56-59; M. ECKHOLT, "Pentekostalismus: Eine neue «Grundform» des Christseins", en T. Kessler/A.-P. RethmanN (dir.), Pentekostalismus: Die Pfingstbewegung als Anfrage an Theologie und Kirche (Pustet, Regensburg 2012) 202-225; M. ECKHOLT, "Pentekostalisierung des Christentums? Zur «Rekonfiguration» der religiösen Landkarte in Lateinamerika", en Stimmen der Zeit 138 (2013) 507-520. - Von diesem neuen „Stil“ des Christentums ist auch in der evangelischen Missionstheologie die Rede: vgl. Th. SundermeIER, Mission - Geschenk der Freibeit. Bausteine für eine Theologie der Mission (Verlag Otto Lembeck/Frankfurt a.M. 2005), 150-153. 
abgesprochen werden und sich Glaube "exkarniert", nicht mehr mit der alltäglichen, ambivalenten, widersprüchlichen, konfliktiven Lebensrealität der Menschen verbunden ist. Das ist eine Gefahr, die manche Pfingstkirchen laufen, aber es ist auch der Spiegel, den die Pfingstbewegung den etablierten christlichen Kirchen vorhält: den Mut zu haben zu Reformen, wenn Institutionen nicht mehr tragen und Menschen heute nicht mehr helfen, lebendige Glaubensformen auszubilden. Die "Entdeckung der anderen", für die aus katholischer Perspektive das 2. Vatikanische Konzil steht und für die in den Befreiungstheologien der Länder des Südens neue Hermeneutiken entwickelt worden sind, ist noch lange nicht abgeschlossen, und sie ist gerade angesichts des neuen Phänomens des "Pentekostalismus" eine gemeinsame Herausforderung der christlichen Kirchen ${ }^{33}$.

Für die katholische Kirche wird dies bedeuten, aus der Einsicht in die fehlgehenden Prozesse der "Entdeckung der anderen" in der Geschichte und in der Erinnerung an die befreienden Traditionen christlichen Glaubens aus der versöhnenden Kraft des Evangeliums in eine neue Weite des KircheSeins hineinzuwachsen. Mit dem Pontifikat von Franziskus, dem argentinischen Papst "vom Ende der Welt", ist dabei die Hoffnung gegeben, dass die katholische Kirche 50 Jahre nach dem Erneuerungs- und Reformprozess, für den das 2. Vatikanische Konzil (1962-1965) steht, diese Reform "realisiert". Papst Franziskus stellt seit Beginn seines Pontifikats die Reformbewegung, die das 2. Vatikanische Konzil für die katholische Kirche bedeutet, in das Zentrum seiner Ansprachen und Predigten ${ }^{34}$. Das ist

33 Auch das Dokument "Vom Konflikt zur Gemeinschaft" weist auf den Pentkostalismus hin: $\mathrm{Zu}$ dieser Relektüre der Tradition wird es gehören, die Perspektiven der neuen pentekostalen und charismatischen Bewegungen aufzugreifen und im Sinn einer lebendigen Erinnerung an die Reformation auch notwendige Reformprozesse in den christlichen Kirchen anzustoßen. So heißt es im Bericht: "Seit mehr als hundert Jahren verbreiten sich pfingstlerische und charismatische Bewegungen über die ganze Welt. Diese kraftvollen Bewegungen haben neue Schwerpunkte gesetzt, die manche der alten konfessionellen Kontroversen obsolet erscheinen lassen. Die Pfingstbewegung ist in vielen anderen Kirchen in Form der charismatischen Bewegung präsent; sie schafft neue Gemeinsamkeiten und Gemeinschaften über konfessionelle Grenzen hinweg. Damit eröffnet diese Bewegung neue ökumenische Möglichkeiten und schafft gleichzeitig zusätzliche Herausforderungen, die bei der Gestaltung des Reformationsgedenkens 2017 eine wichtige Rolle spielen werden” (S. 18, Nr. 14).

34 Vgl. M. Eскнодт, "Ein Papst des Volkes: Die lateinamerikanische Prägung von Papst Franziskus“, en Theologisch-praktische Quartalschrift 163 (2015) 4-19; 
eine Kirche "im Aufbruch" (vgl. EG 20-23), "semper reformanda"35, ein "Feldlazarett" ${ }^{6}$ und keine "feste Burg", eine Kirche, der es um "confessio" geht, aber nicht als Abgrenzung vom anderen, sondern im Sinn eines lebendigen Glaubens und gemeinsamen Christuszeugnisses "im

Papst Franziskus, Apostolisches Schreiben Evangelii Gaudium über die Verkündigung des Evangeliums in der Welt von heute, editado por el Sekretariat der Deutschen Bischofskonferenz (Bonn 2013).

35 Der argentinische Theologe Carlos Galli weist auf den mehrfachen Gebrauch dieses Begriffs durch Papst Franziskus hin: „Pablo VI y Francisco: La alegría de Cristo”, en Istituto Paolo VI Notiziario 72 (2016) 43-71, 70: "En 2015 Francisco empleó dos veces la frase Ecclesia semper reformanda, a la que se han dedicado varios estudios en una obra colectiva reciente78. En una ocasión se refirió a la reforma de la Iglesia y en otra a la de la Curia romana. En el V Congreso de la Iglesia italiana en Florencia explicó que «la reforma de la Iglesia - y la Iglesia es semper reformanda - es ajena al pelagianismo. Ella no se agota en el enésimo proyecto para cambiar las estructuras. Significa en cambio injertarse y radicarse en Cristo, dejándose conducir por el Espíritu. Entonces todo será posible con ingenio y creatividad». Allí puso el acento en la obra renovadora del Espíritu. Luego, en su saludo de Navidad a los miembros de la Curia, afirmó que «la reforma seguirá adelante con determinación, lucidez y resolución, porque Ecclesia semper reformanda». Esa consigna expresa la necesidad de la renovación permanente y el cambio en cada momento histórico. Implica dar un paso hacia adelante en el camino de la conversión personal, comunitaria y estructural hacia la plena comunión con Cristo en el amor: «En la historia de la Iglesia católica los verdaderos renovadores son los santos. Ellos son los verdaderos reformadores, los que cambian, transforman, llevan adelante y resucitan el camino spiritual»". Und: "Francisco invita a todo el Pueblo de Dios y a todos en el Pueblo de Dios a continuar «la reforma misionera todavía pendiente» (LS 3). Su proyecto se cifra en la «conversión misionera» (EG 30) o «la reforma de la Iglesia en salida misionera» (EG 17), Evangelii gaudium emplea la frase «pastoral en conversión» (EG 25-33), la que recrea la propuesta de Aparecida sobre la conversión pastoral y la renovación misionera (DA 365-372). Un «estado permanente de misión» (EG 25) exige reformar las estructuras «para que se vuelvan más misioneras» (EG 27), incluyendo la conversión del Papado con las estructuras centrales de la Iglesia (EG 32). La Ecclesia semper reformanda es una Ecclesia in statu conversionis e in statu missionis". PAPST Franziskus, Der Name Gottes ist Barmber:igkeit: Ein Gespräch mit Andrea Tornielli (Kösel, München 2016); vgl. dazu: http://www.katholisch.de/aktuelles/aktuelleartikel/franziskus-die-kirche-ist-ein-feldlazarett; vgl. http://de.radiovaticana.va/ news $/ 2015 / 02 / 05 / \mathrm{fr} \% \mathrm{C} 3 \%$ BChmesse_die_kirche_ist_ein_feldlazarett/1121657: „Das ist die Mission der Kirche, einer Kirche, die heilt und pflegt. Ich habe in der Vergangenheit von der Kirche als einem Feldlazarett gesprochen. So ist es! Es gibt so viele Verletzte! Es gibt so viele Menschen, die der Pflege bedürfen! Das ist der Auftrag der Kirche: Pflegt die Wunden des Herzens, öffnet Gott die Tore, denn Er ist gut und vergibt alles, weil Gott ein zärtlicher Vater ist. Gott wartet immer auf uns!“ 
Dienst an der Einheit und am Frieden der Welt" ${ }^{37}$. Das ist dann eine Kirche, die "missionarisch" ist in genau dem Sinn, dass sie sich selbst in der "Entdeckung der anderen" je neu zu Jesus Christus "bekehrt". Der argentinische Papst vom "Ende der Welt" stößt 50 Jahre nach dem 2. Vatikanischen Konzil einen Prozess der Erinnerung an, der die heute leitenden Horizonte des Reformationsgedenkens - die gemeinsame Erinnerung und die internationale Perspektive -, die im Ursprung der Reformation zusammengehören, aber im Zeitalter der "Konfessionalisierung" auseinandergefallen sind, wieder zusammenführt. Die "Ekklesiogenese" zu der er aufruft, betrifft viele der Momente, für die die Reformation steht: die volkskirchliche Liturgie, der je neue Rückbezug auf die biblischen Texte, das gemeinsame Priestertum und damit die Subjektwerdung der Glaubenden, Momente, die in den verschiedenen befreiungstheologischen Entwürfen und neuen Formen des KircheSeins in den letzten 50 Jahren lebendig geworden sind. In den Prozess der Ekklesiogenese und damit des "healing of memories" bezieht Papst Franziskus die Symbolisierung der sichtbaren Einheit der christlichen Kirchen im Amt des Papstes ein, der Bischof von Rom und in diesem Sinn "Bruder" ist und dessen Amt im Dienst des Friedens und des "universalen Humanismus" steht, "der in Jesus Christus als dem neuen und letzten Adam grundgelegt ist" (1 Kor 15,45) ${ }^{38}$. Damit ruft er Papst Hadrian VI. (Pontifikat von 1522 bis 1523) in Erinnerung und seine Bitte um Verzeihung, die er angesichts der Verwerfungen Luthers in seiner Botschaft an den Reichstag in Nürnberg vom 25. November 1522 ausgedrückt hat und die am 3. Januar 1523 in Nürnberg verlesen wurde $^{39}$. Das ist ein starkes und symbolkräftiges Zeichen der Ökumene

\footnotetext{
W. KASPER, Martin Luther ..., 51.

W. KASPER, Martin Luther ..., 51.

Das Dokument „Vom Konflikt zur Gemeinschaft“ bezieht sich auf die Bitte um Verzeihung, die Papst Hadrian VI. in seiner Botschaft an den Reichstag in Nürnberg am 25. November 1522 ausgedrückt hat, und an die erst im 20. Jahrhundert die Päpste wieder anknüpfen. Paul VI. wird bei der Eröffnungsrede des Zweiten Vatikanischen Konzils zur zweiten Sitzung die "getrennten Brüder des Ostens um Verzeihung" bitten (Nr. 234, S. 92), und Johannes Paul II. wird anlässlich der Feiern des Heiligen Jahres 2000 um Verzeihung bitten. In seiner Enzyklika "Ut unum sint" (1995) hat er vor allem auch darauf hingewiesen, dass gerade das Amt des Bischofs von Rom Anlass für Konflikt und Ausgrenzung gewesen ist. Und er sagt: "Soweit wir dafür verantwortlich sind, bitte ich mit meinem Vorgänger Paul VI. um Verzeihung” (Nr. 235, S. 93). - vgl. dazu: V. Leppin, Die fremde Reformation..., 166.
} 
86 | Margit Eckholt

im Jahr des Gedenkens der Reformation, Ausdruck dessen, dass das konfessionelle Zeitalter "unwiderruflich vorbei und jeder Versuch, es auf den Ruinen der Vergangenheit wiederzubeleben, zum Scheitern verurteilt ist" ${ }^{\prime 40}$, so Kardinal Walter Kasper in seinen Überlegungen zu Martin Luther in ökumenischer Perspektive.

Das sind Wege in den Ursprungsgrund des Christentums, das Evangelium des Friedens, der Gnade, der Rechtfertigung und Befreiung, in dessen Tiefe die "Entdeckung der anderen" begründet ist: mich je neu von Christus aus meiner Selbstbezogenheit zu befreien, zum anderen, zur anderen hier - im Dienste des Lebens: miteinander. Das ist dann das "Christusfest", das nicht nur 2017 zu feiern ist, sondern alltäglich, in der Unverhofftheit des Einbruchs des "anderen" - Jesus Christus - in mein Leben.

40 W. Kasper, Martin Luther. .., 38. 